\title{
Capillarylike Fluctuations at the Interface of Falling Granular Jets
}

\author{
Yacine Amarouchene, ${ }^{*}$ Jean-François Boudet, and Hamid Kellay \\ Université Bordeauxl, Centre de Physique Moléculaire Optique et Hertzienne, \\ CNRS UMR 5798 and 351, cours de la liberation 33405 Talence, France \\ (Received 18 January 2008; published 27 May 2008)
}

\begin{abstract}
We study the interface fluctuations of a granular jet falling under gravity and show that for small scales they are the analog of the thermally induced capillary waves. Experimental results from radial height and velocity fluctuations, static correlation functions and capillary ripple velocities allow us to estimate a granular surface tension. The ultralow interfacial tensions measured (of the order of $100 \mu \mathrm{N} / \mathrm{m}$ ) can be rationalized using a simple model.
\end{abstract}

DOI: 10.1103/PhysRevLett.100.218001

PACS numbers: 45.70. $-\mathrm{n}, 47.20 . \mathrm{Dr}, 47.50 . \mathrm{Gj}$

The free interface between any two fluids, like that between a liquid and its vapor is not as smooth and mirror like as it appears on a macroscopic scale. In fact, thermal motion gives rise to a rough interface that can be described in terms of thermally excited capillary waves [1,2]. These thermal capillary waves have been studied extensively in molecular fluids using surface light [3] and x-ray scattering [4]. Recently, they have also been visualized in real space in a phase separated colloid polymer mixture [5], where excellent agreement with capillary-wave theory was found. In such colloidal systems the surface tension scales as $k_{B} T / d^{2}$, where $d$ is the particle size and $k_{B} T$ the thermal energy, leading to ultralow values for $\sigma$ (down to $10^{-6} \mathrm{~N} / \mathrm{m}$ ). When the size of the entities become large enough that Brownian motion is irrelevant $(d \gg 1 \mu \mathrm{m})$, we leave the colloidal domain for the granular one and intrinsically different properties emerge.

While, in many instances, the macroscopic behavior of granular materials may seem liquidlike, gaslike, or solidlike, granular materials remain more complex to understand than their molecular counterparts [6]. In particular, granular flows may exhibit intrinsic peculiar collective phenomena [6], such as segregation [7], pattern formation, clustering [6], and even shocks waves [8,9] shear instabilities [10], and turbulent like behavior [11]. Since the thermal energy becomes irrelevant, a steady state can only be reached through an external excitation.

Recently, a granular analog of the well-known RayleighTaylor instability renewed the interest in the relevance of an effective granular surface tension [12]. Tapping repeatedly a horizontal flat plate initially covered with a monolayer of fine powder particles, Duran has observed powder "droplets" that can be described using the equivalent of the Laplace law and a surface tension $(\sigma \sim 25 \mu \mathrm{N} / \mathrm{m}$ for $20 \mu \mathrm{m}$ grains) whose origin is the flow of interstitial air between grains. In addition to these observations, a capillary like "clustering" instability have been also evidenced during the free fall of granular jets either in air [13] or in a quiescent liquid bath [14]. The granular flow out of a circular nozzle starts out spatially uniform and then, further downstream, breaks up into well-defined "clusters, blobs, and drops" as may happen for ordinary liquids. Thus, we may ask whether an effective surface tension is relevant in the general context of granular physics. This Letter presents experimental results from a study of dry granular jets falling from a funnel shaped container. Figure 1 represents a typical falling granular jet at two different distances from the outlet. Note that the interface is not smooth. We show that this roughness is the analog of the thermally induced capillary waves at an interface. An analysis of the statics and the dynamics of these fluctuations allows for a direct estimate of a granular surface tension.

Our system consists simply of a granular jet falling from a funnel shaped container that remains open to avoid intermittent hourglass ticking effects [15]. The mass flux for this setup is controlled by the diameter $D=12 \mathrm{~mm}$ of the funnel outlet. All experiments have been performed with quasi monodisperse $d=159 \mu \mathrm{m}$ spherical glass beads (Potters Ballotini density $\rho_{b}=2500 \mathrm{~kg} \cdot \mathrm{m}^{-3}$ ). To minimize the effects of humidity the samples were dried in an oven.

Since we use a broad diffuse light, the granular jet (liquid phase) appears dark and the grain-poor region (gas phase) appears brighter. The intensity distribution of transmitted light, $I(z, r, t)$ is recorded using a fast camera (5000 frames per second and $70 \mu \mathrm{s}$ exposure times) at a certain time $t$, with $z$ being the vertical (along the interface, in gravity direction) and $r$ the radial components. The
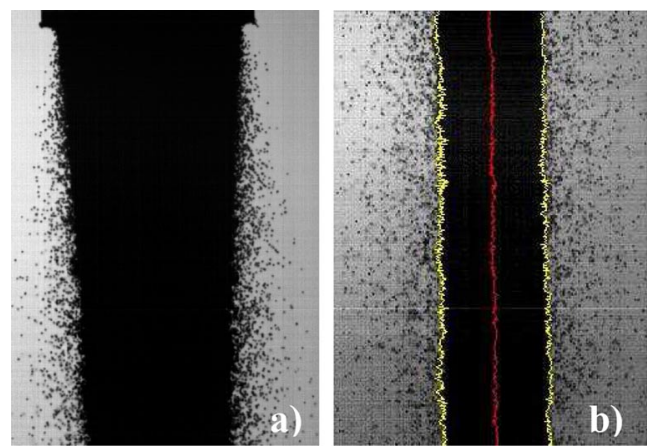

FIG. 1 (color online). Snapshots of the granular jet close to the outlet (a) and $26 \mathrm{~cm}$ downstream (b). 
objective lens used allowed us to reach length scales smaller than $d / 4$. Relying on the concept of the Gibbs dividing surface [2], we can define an interface position $h(z, t)$ such that along a horizontal segment of length $L_{r}$ whose origin is the center of the jet, the total intensity can be written as $\int_{0}^{L_{r}} I(z, r, t) d r=I_{\mathrm{gas}}(z) h(z, t)+I_{\mathrm{liq}}(z)\left[L_{r}-\right.$ $h(z, t)]$ where $I_{\text {gas }}(z)$ and $I_{\text {liq }}(z)$ are the average intensities in the gas and liquid phase, respectively, and are taken to be functions of $z$ to account for inhomogeneous lighting and objective lens properties. The resulting "height" function $h(z, t)$ follows the contour of the jet quite accurately (yellow line in Fig. 1(b).) [16].

We estimate the volume fraction $\Phi$ at the exit of the funnel using mass conservation through the relation $\Phi=$ $4 Q /\left(\rho_{b} V_{0} \pi D^{2}\right)$, with $Q$ being the mass flux and $V_{0}$ the mean flow velocity at the outlet. The jet density is then simply $\rho=\rho_{b} \Phi$. The jet radius decreases along the vertical position $z$ as $r_{0}(z) \sim \frac{D}{2}\left(1+\frac{z}{D}\right)^{-1 / 4}$ which seems to be compatible with the incompressible hypothesis at least for the range of vertical scales probed here. The scaling is slightly different from our previous measurements because it depends on the grain size and the funnel diameter [17]. The volume fraction is almost constant along the jet and is close to the typical "loose packing" value commonly reported for granular flows $\Phi \sim 0.5$.

Velocity measurements are carried out using particle image velocimetry (PIV) through the calculation of the cross-correlation between two snapshots of the particle field separated by a small time interval. This technique allows to measure the velocity field (mean velocity $V$ and its fluctuations). In particular, the velocity fluctuations allow us to compute the granular or effective temperature defined as $T=\frac{\delta V^{2}}{2}$ by analogy with kinetic theories. Figure 2(a) displays a typical radial cut of the magnitude of the velocity and its standard deviation. The velocity and the standard deviation very near the jet are well defined, with $V$ decreasing slightly as $r$ increases. The velocity $V$ along the interface [see Fig. 2(b)] turns out to be slightly smaller than what is expected for a free fall $V \simeq$ $\sqrt{2 g z+V_{0}^{2}}, g$ being the gravity constant $g=9.81 \mathrm{~m}$. $\mathrm{s}^{-2}$ and $V_{0} \simeq 0.25 \mathrm{~m} \cdot \mathrm{s}^{-1}$. One must note that the influence of air drag becomes more important as $z$ increases leading to a slightly higher deviation of the jet surface velocity from the free fall value. In fact, the measured values are bounded from above by the free fall velocity and from below by the velocity of a single particle under the action of gravity and Stokes drag [18]. The velocity standard deviation $\delta V$ very near the surface seems to follow a linear dependence versus $z$. Thus, the temperature $T(z) \simeq 1 / 2\left(\delta V_{0}+A z\right)^{2}$ is roughly quadratic with the distance (with $A=0.41 \mathrm{~s}^{-1}$ ) with a constant injected temperature at the outlet (for $z \ll D$ we have $\delta V_{0} \sim 0.025 \mathrm{~m}$. $\mathrm{s}^{-1}$ ). Note that the velocity statistics along the interface are Gaussian [see Fig. 2(c)]. This allows us to define a standard deviation unambiguously.

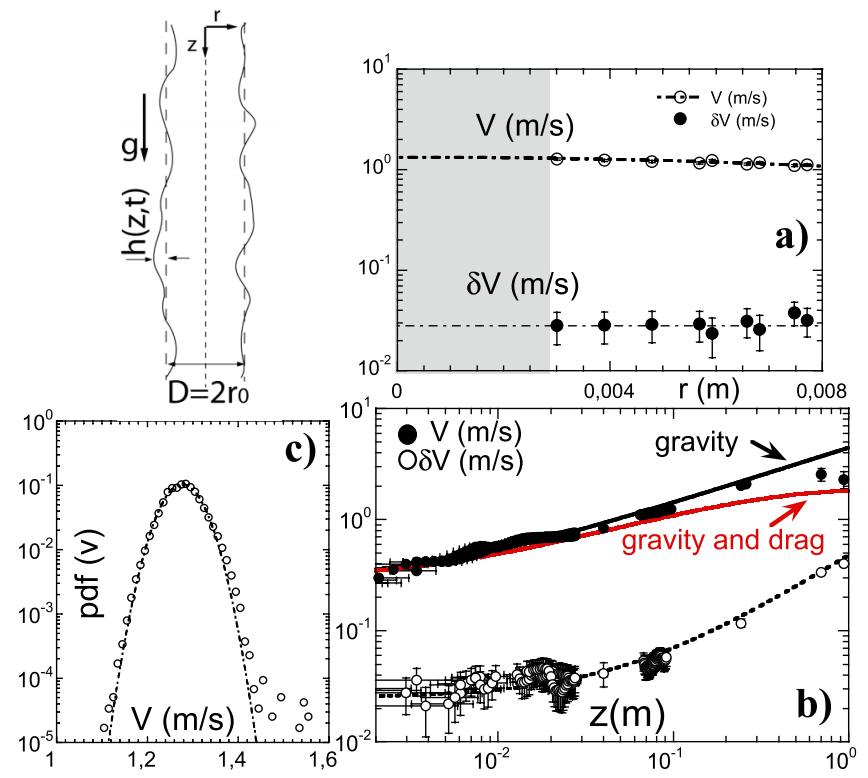

FIG. 2 (color online). (a) $V(r)$ and $\delta V(r)$ (gray area: jet; dotted lines: guides to the eye) $z=10 \mathrm{~cm}$. (b) $V(z)$ and $\delta V(z)$ at the interface of the jet. Black line: free fall. Red line: Stokes Drag force $F_{D}=3 \pi \eta d V$. For $z>0.1 \mathrm{~m}$, error bars are smaller than the points for $\delta V(z)$. (c) $\operatorname{PDF}(V)$ at the jet interface for $z=$ $10 \mathrm{~cm}$.

Let us now examine the interfacial fluctuations. The probability distribution $\operatorname{PDF}(h)$ of the radial height fluctuations $h$ is Gaussian as illustrated in Fig. 3(a) for different vertical positions (the mean jet radius has been substracted). The radial height amplitude standard deviation measured along the jet $\left\langle\xi_{\perp}^{2}\right\rangle^{1 / 2}$ shown in Fig. 3(b) displays an interesting nonmonotonic behavior with a sharp maximum at a distance close to the funnel outlet diameter. Thus most radial height amplitude fluctuations seem to be located close to the outlet in agreement with previous measurements [13].

In order to check whether a surface tension is relevant or not, we examine the propagation of capillary waves. Indeed, in the inviscid limit, modes with wavelengths smaller than the jet circumference $2 \pi r_{0}$ are stable and propagative with a phase speed $c\left(q r_{0}\right)=\sqrt{\frac{\sigma}{\rho r_{0}}}\left[\frac{1}{q r_{0}} \frac{I_{1}\left(q r_{0}\right)}{I_{0}\left(q r_{0}\right)}\left(1-\left(q r_{0}\right)^{2}\right)\right]^{1 / 2}$
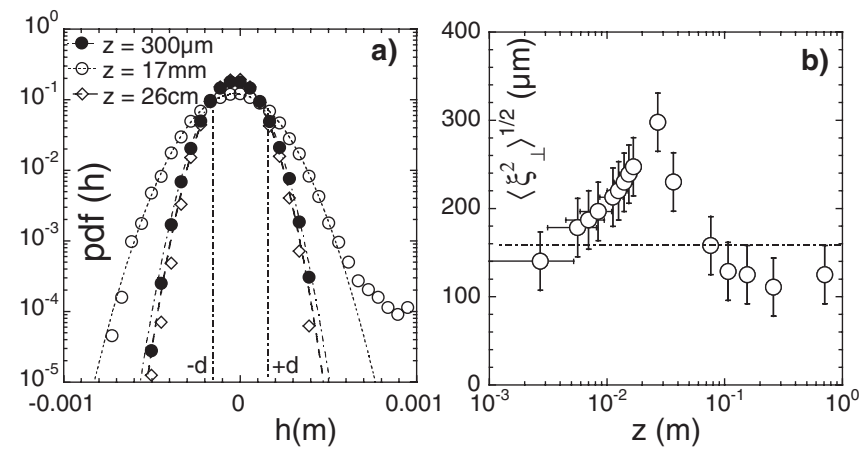

FIG. 3. (a) $\operatorname{PDF}(h)$. lines: Gaussian fits; vertical dashed lines: $\pm 1 d$. (b) $\left\langle\xi_{\perp}^{2}\right\rangle^{1 / 2}$ versus $z$. horizontal line: $1 d$. 
[19] $I_{1}$ and $I_{0}$ being modified Bessel functions of the first kind. Here $\lambda=2 \pi / q$ is the wavelength of the capillary excitation of amplitude $h$ and wave number $q$ (see inset to Fig. 4 for details). In other words, surface tension may give rise to stable small scale dispersive modes with velocities greater than the mean velocity of the jet. In order to carry this through, one must select a single wavelength. The radial height profile is not smooth and shows roughness at different length scales like the surface of molecular fluids where capillary fluctuations of different wavelengths are excited by thermal motion. In order to focus on a particular wavelength, one can Fourier bandpass the signals at that particular wavelength as shown in the inset to Fig. 4 for $q r_{0}=3.37$. The measured velocity for this example is $2.20 \pm 0.01 \mathrm{~m} / \mathrm{s}$ while the measured mean velocity is $2.144 \pm 0.001 \mathrm{~m} / \mathrm{s}$ at this location. However, for Fourier bandpassing one has to localize regions with high monochromatic content (it is what we have done on a small sample of the signal in the inset to Fig. 4). This reduces the accuracy in the measurement of the velocity shift since the amount of statistics is considerably reduced. An alternative to Fourier transforms that improves the accuracy in the measurement of the velocity shift that we seek to analyze is wavelet analysis [20].

Wavelets allow for an analysis of extremely local behavior (since they are compactly supported) without windowing worries and with negligible bias induced from global behavior. Wavelets are widely used in several areas (fractal properties of a signal, denoizing, compression...). The wavelet transform is a space-scale analysis which consists of expanding signals in terms of wavelets that are constructed from the analyzing wavelet $\psi$ by means of dilations and translations. The wavelet transform of our profiles (at each time $t) h(x, t)$ is defined as $T_{\psi}\left(x_{0}, a, t\right)=$ $\frac{1}{a} \int_{-\infty}^{+\infty} h(x, t) \psi\left(\frac{x-x_{0}}{a}\right) d x$, where $x_{0}$ and $a$ are the space and

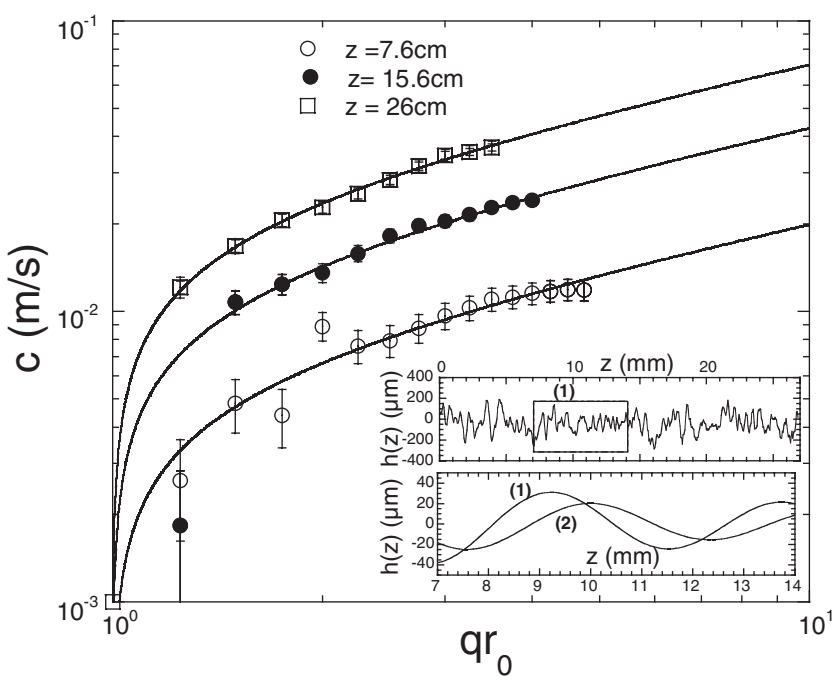

FIG. 4. $c\left(q r_{0}\right)$. lines: fits to the theoretical expression. inset: top profile: unfiltered profile; bottom profiles: band-pass filtered at different times $(\Delta T=2 / 5000 \mathrm{~s})$. scale parameters, respectively. In this work, we used the second Daubechies function: $\psi^{(2)}(x)$ which presents good localization properties [20]. The information needed to estimate the phase speed of the stable propagative modes requires a measurement of the distance traveled during a fixed small time interval for each selected mode (see inset of Fig. 4) with excellent precision. This is performed through the detection of the peak of the spatial crosscorrelation function of two filtered profiles at different successive times. The phase speeds measured (minus the mean advection velocity $V$ ) are shown in Fig. 4. along with the theoretical fit for different $z$. Since the procedure requires a very weak variation of the mean velocity on the scale of the observation window (typically $1 \mathrm{~cm}$ ), only results far downstream are shown. Our results show that small structures $\left(q r_{0}>1\right)$ move with a velocity slightly larger than the jet's mean velocity in agreement with recently published numerical results [21]. The typical phase speeds $\sqrt{\frac{\sigma}{\rho r_{0}}}$ obtained from the fit to the theoretical expression are in the $\mathrm{cm} / \mathrm{s}$ range. The surface tension estimated from our phase speed measurements at locations $z=7.6,15.6,26 \mathrm{~cm}$ are, respectively, 217, 836, and $1994 \mu \mathrm{N} / \mathrm{m}$.

To pursue the analogy with capillary waves, the next step is to look for an estimate of the granular surface tension through the phenomenological relation $\sigma=$ $\frac{k_{B} T}{\left(2 \pi\left\langle\xi_{\perp}^{2}\right\rangle\right)} \log \left(\frac{L}{d}\right)[2](L \sim 100 d$ being the window size $)$. The granular temperature $\left(k_{B} T \equiv \frac{1}{2} m \delta V^{2}\right)$ and $\left\langle\xi_{\perp}^{2}\right\rangle$ have been determined separately as explained above. The results that are displayed in Fig. 5 show that the surface tension parameter $\sigma$ is almost constant for $z \ll D$ with a value close to $35 \mu \mathrm{N} / \mathrm{m}$. For larger distances, $\sigma$ increases as $z$ increases. It reaches $1 \mathrm{mN} / \mathrm{m}$ at $26 \mathrm{~cm}$ downstream. The surface tensions measured using temperature and $\left\langle\xi_{\perp}^{2}\right\rangle$ measurements turn out to be remarkably close to the values estimated from the phase speeds as shown in Fig. 5.

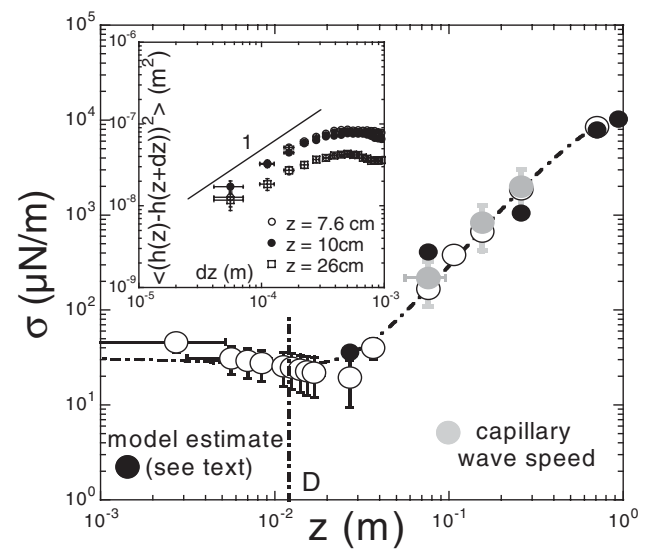

FIG. 5. $\sigma$ vs $z$. White circles: from fluctuations. Gray points: from phase speeds. Black points: estimates of $\sigma$ from our model. vertical dotted line: $D$; other dotted line: guide to the eye. Inset: $S_{2}(d z)$ versus $d z$. 
More information on the physical properties of the interface can also be obtained by looking at the second order structure function $S_{2}(d z)=\left\langle(h(z+d z)-h(d z))^{2}\right\rangle$ [22]. The radial height correlation power spectrum $\langle h(q) h(-q)\rangle \propto \frac{k_{B} T}{\sigma q^{2}}$ predicted for interfaces implies that $S_{2} \sim d z$. The inset of Fig. 5. shows that a scaling law consistent with $S_{2} \sim d z$ is observed for scales smaller than a typical scale $\xi_{\|}$of the order of a few grain diameters. At larger scales $d z>\xi_{\|}, S_{2}(d z)$ flattens. One must note that both $\xi_{\perp}$ and $\xi_{\|}$are of the order of few grain diameters in sharp contrast to the molecular case for which a clear separation of scales exist (for systems under gravity $\xi_{\|} \sim$ $10^{-3} \mathrm{~m}$ is the capillary length and $\xi_{\perp} \sim 10^{-10} \mathrm{~m}$ ).

What is the origin of the surface tension we measure? If we assume that the air within the jet and the air outside it but adjacent to the surface travel at different velocities, a pressure difference $\Delta P$ would exist between the outside and the inside of the jet. Previous measurements and numerical simulations show that there is a significant air entrainment induced by the free falling streams of grains [23]. In our case, the velocity $V$ measured along the interface displayed in Fig. 2(b). shows that grains close to the surface of the jet go faster than a single grain, subject to stokes drag in an unbounded fluid, confirming this entrainment. At the center of the jet, we may assume that the air is entrained with a velocity close the free fall velocity as has been suggested previously [23] while the air velocity in the vicinity of the surface is given by the surface velocity of the jet. Thus there is a small but measurable difference between the center velocity $V c=\sqrt{2 g z+V_{0}^{2}}$ and the interface velocity $V$. One can estimate a positive pressure difference between the outside and the inside of the jet using Bernoulli theorem: $\Delta P \approx \frac{\rho_{\text {air }}}{2}\left(V_{c}^{2}-V^{2}\right), \quad \rho_{\text {air }} \sim$ $1 \mathrm{~kg} / \mathrm{m}^{3}$ being the air density. This pressure difference would give rise to an inward pointing force at the surface of the jet. A surface tension can be estimated by the relation $\sigma \approx \Delta P r_{0}(z)$, involving the small depression $\Delta P$, which induces an effective cohesion due to the air pressure difference. The surface tensions estimated using this simple model are very close to the values measured using the two other methods as shown in Fig. 5.

Usually, the importance of surface tension forces versus gravitational forces and inertial ones are estimated using the Bond number, $\mathrm{Bo}=\frac{\rho g r_{0}^{2}}{\sigma}$, and the Weber number, $\mathrm{We}=\frac{\rho U^{2} r_{0}}{\sigma}$. The low values of the surface tension reported in this Letter imply that Bo and We at the scale of the jet radius $r_{0}$ are in the $10^{2}-10^{6}$ range which is much smaller than would be expected from traditional cohesive models, e.g., from liquid bridging or electrostatic effects. Our results suggest that for large scale phenomena the surface tension forces are excessively weak which may allow one to consider granular fluids as model zero surface tension fluids as recently proposed [24]. However, at small scales these capillary forces can be sufficiently strong and may play an important role.
In conclusion, our results suggest that capillary effects can be observed at the interface of granular jets. Experimental evidence of the analog of thermal capillary waves through velocity measurements of capillary ripples and a simple model allows us to estimate a surface tension for granular materials. This opens the way to a better understanding of small scale interfacial phenomena in these widely spread materials.

The research has been supported by the "Conseil Regional d'Aquitaine" through Grant no. 2006111101035.

*y.amarouchene@cpmoh.u-bordeaux1.fr

[1] L. Mandelstam, Ann. Phys. (Berlin) 41, 609 (1913).

[2] J.S. Rowlinson and W. Widom, Molecular Theory of Capillarity (Dover, New York, 2002).

[3] J. Meunier, Liquids and Interfaces, edited by J. Charvolin, J. F. Joanny, and J. Zinn-Justin (North-Holland, New York, 1988), p. 327.

[4] B. M. Sanyal et al., Phys. Rev. Lett. 66, 628 (1991).

[5] D. G. A. L. Aarts, M. Schmidt, and H. N. W. Lekkerkerker, Science 304, 847 (2004).

[6] H. M. Jaeger, S. R. Nagel, and R. P. Behringer, Rev. Mod. Phys. 68, 1259 (1996).

[7] I. Zuriguel et al., Phys. Rev. Lett. 95, 258002 (2005).

[8] E. C. Rericha et al., Phys. Rev. Lett. 88, 014302 (2001).

[9] Y. Amarouchene and H. Kellay, Phys. Fluids 18, 031707 (2006).

[10] D. J. Goldfarb, B. J. Glasser, and T. Shinbrot, Nature (London) 415, 302 (2002).

[11] H. Kellay, Y. Amarouchene, and J.-F. Boudet, Phys. Fluids 19, 078104 (2007).

[12] J. Duran, Phys. Rev. Lett. 87, 254301 (2001).

[13] M.E. Möbius, Phys. Rev. E 74, 051304 (2006).

[14] M. Nicolas, Phys. Fluids 14, 3570 (2002); U. Schaflinger and G. Machu, Chem. Eng. Technol. 22, 617 (1999).

[15] X.-L. Wu et al., Phys. Rev. Lett. 71, 1363 (1993).

[16] van der Waals estimate of the interface position using tanh fits give similar results.

[17] J.-F. Boudet et al., J. Fluid Mech. 572, 413 (2007).

[18] The red line comes from the integration of $V(t)=V_{T}(1-$ $\left.\exp \left(-18 \eta t / \rho d^{2}\right)\right)$ with a terminal velocity $V_{T}=g d^{2}(\rho-$ $\left.\rho_{\text {air }}\right) / 18 \eta_{\text {air }} \sim 1.86 \mathrm{~m} / \mathrm{s}$.

[19] S. Chandrasekhar, Hydrodynamic and Hydromagnetic Stability (Dover, New York, 1981); V. G. Levich, Physicochemical Hydrodynamics (Dekker, New York, 1962).

[20] A. Arneodo et al., Ondelettes, multifractales et turbulences (Diderot éditeur, Paris, 1995); D. B. Percival and A. T. Walden, Wavelet Methods for Time Series Analysis (Cambridge University Press, Cambridge, England, 2006).

[21] A. Alvarez, E. Clement, and R. Soto, Phys. Fluids 18, 083301 (2006).

[22] S. K. Sinha et al., Phys. Rev. B 38, 2297 (1988).

[23] K. Ogata, K. Funatsu, and Y. Tomita, Powder Technol. 115, 90 (2001); T. Uchiyama and M. Naruse, Chem. Eng. Sci. 61, 1913 (2006); Z. Liu, P. Cooper, and P. W. Wypych, Part. Sci. Technol. 25, 357 (2007).

[24] X. Cheng et al., Phys. Rev. Lett. 99, 188001 (2007). 